\title{
G Protein-coupled Receptor 30 Regulates Trophoblast Invasion And Its Deficiency Is Associated With Preeclampsia
}

\section{Running title: Preeclampsia is associated with compromised G Protein- coupled Receptor 30 expressison, which is involved in regulating trophoblast invasion}

Chao Tong1, 2, Xiang Feng ${ }^{1,2}$, Jun Chen ${ }^{1,2}$, Xingchen Qi ${ }^{2}$, Liyuan Zhou ${ }^{1,2}$, Shuming $\mathrm{Shi}^{1,2}$, Kamana KC ${ }^{1,2}$, Joanna L. Stanley ${ }^{3}$, Philip N. Baker ${ }^{2,3}$, Hua Zhang ${ }^{1,2 *}$

${ }^{1}$ Department of Obstetrics and Gynecology, The First Affiliated Hospital of Chongqing Medical University, Chongqing 400016, People's Republic of China ${ }^{2}$ Canada - China New Zealand Joint Laboratory of Maternal and Fetal Medicine, Chongqing Medical University, Chongqing 400016, People's Republic of China, 3 Liggins Institute, University of Auckland, Auckland, New Zealand

This work was supported by grants from the National Natural Science Foundation of China (No. 81170585, 81100444, 81370732), Chongqing Science and Technology Commission (No. 2011BB5121) and Chongqing Municipal Health Bureau (No.2011-2046).

NONE disclaimers

*Corresponding Author: Hua Zhang, MD, Ph.D.

Department of Obstetrics and Gynecology, The First Affiliated Hospital of Chongqing Medical University, Canada - China -New Zealand Joint Laboratory of Maternal and Fetal Medicine, Chongqing Medical University, No. 1 Youyi Road, Yuzhong District, Chongqing 400016, People's Republic of China.

Tel: +86-23-89011102; Fax: +86-23-89011082;

Email: zh2844@gmail.com

Word count: $3791 \quad$ Number of Tables: $1 \quad$ Number of Figures: 7 


\begin{abstract}
Preeclampsia (PE) is known to be associated with reduced circulating levels of estrogen. The effects of estrogen in PE are normally mediated by the classical estrogen receptors (ERs). Intriguingly, a novel estrogen receptor, G-protein coupled receptor 30 (GPR30), has been recently found to play an important role in several estrogenic effects. However, the mechanisms by which GPR30 may mediate the development of PE remain unknown. We observed that the expression of GPR30 in placental trophoblast cells is lower in preeclamptic placentas compared with normotensive controls. We then investigated the role of GPR30 in trophoblast cell invasion by utilizing placental explants and the immortalized human trophoblast cell line (HTR8/SVneo). The selective GPR30 agonist G1 and a general estrogen receptors agonist 17- $\beta$-estradiol (E2), both improved trophoblast cells invasion by up-regulating MMP9 expression and the PI3K-Akt signaling pathway. This effect was abolished by a selective GPR30 inhibitor G15, implying that GPR30 may be involved in regulating trophoblast invasion, and that deregulation of this receptor may result in the development of PE. The present study suggests that GPR30 is a critical regulator of trophoblast cell invasion, and as such may be a potential therapeutic interventional target for $\mathrm{PE}$ and other pregnancy complications resulting from impaired trophoblast invasion.
\end{abstract}

Keywords: preeclampsia; G-Protein coupled receptor 30 (GPR30); trophoblast cells; invasion. 


\section{Introduction}

Preeclampsia (PE) is a severe pregnancy-associated disorder, which is characterized by the development of hypertension and proteinuria after 20 weeks of gestation. It complicates $2-7 \%$ of pregnancies globally and significantly contributes to maternal and fetal mortality[1,2]. Its etiology and pathogenesis, however, have not yet been fully elucidated.

Previous studies have shown that defective trophoblast invasion results in shallow placental implantation and inadequate remodeling of the uterine spiral arteries, which in turn leads to hypoperfusion of the placenta and ultimately development of PE and other complications[3-11]. It is clear, therefore, that the maintenance of appropriate trophoblast cells invasion is critical for normal pregnancy. The mechanisms regulating trophoblast invasion, however, are yet to be fully understood.

The G protein-coupled estrogen receptor GPER1, also known as GPR30, was first defined as a novel receptor for estrogen in 2005. Although GPR30 is known to be involved in pathways that are mediated by the classic ERs, its role in mediating rapid estrogen responses in a wide variety of cell types distinguishes it from the other $\operatorname{ERs}[11,12]$.

GPR30 has been intensively studied in estrogen associated cancers, such as breast cancer, endometrial cancer and chorionic carcinoma. Accumulating evidence suggests that GPR30 mediates the effects of estrogen in promoting cancer cell proliferation and invasion[13-17].

Given that trophoblast cells in early pregnancy display a highly invasive phenotype, which is similar to cancer cells[18, 19], and that circulating estrogen levels are significantly elevated during pregnancy, it is possible that estrogen plays a role in regulating trophoblast cell invasion, and that these effects may be mediated by GPR30. These questions, however, are yet to be addressed.

In this study, we firstly aimed to determine the expression patterns of GPR30 in placentas from normal and PE complicated pregnancies. We then aimed to study the role of GPR30 in trophoblast cells invasion, as well as, the underlying molecular mechanisms, both of which could offer an insight into the pathogenesis of PE.

\section{Materials and Methods}


This study was approved by the Ethics Committee of Human Experimentation of the First Affiliated Hospital of Chongqing Medical University. Informed consent was received from all subjects.

\section{Study populations}

A total of 41 singleton pregnant women participated in this study. Human placental tissue from first trimester ( 6 and 10 weeks of gestation, $n=5$ in each group) was obtained from 10 healthy women undergoing pregnancy interruption by nonmedical reasons. Fresh term placental tissue was collected from women with preeclampsia (PE group: $n=21$ ) and gestational matched controls (GMCs: normotensive pregnancies without other complications, $n=20$ ); all women were scheduled to undergo caesarean section at the Department of Gynecology and Obstetrics in The First Affiliated Hospital of Chongqing Medical University between January 2013 and May 2014 due to medical need or personal request. The diagnostic criteria of PE was defined according to the guideline of American College of Obstetrics and Gynecology[20]. Namely, systolic blood pressure $\geq 140 \mathrm{~mm} \mathrm{Hg}$ or diastolic blood pressure $\geq 90 \mathrm{~mm} \mathrm{Hg}$, with proteinuria ( $>300 \mathrm{mg}$ protein in 24 hours or a random dip-stick urine which measured $>1+$ protein). All cases of chronic medical disorders such as diabetes mellitus, cardiovascular disease, collagen disorder, chronic renal disease, chronic hypertension, and metabolic diseases were excluded. Subject characteristics are shown in Table 1.

\section{Tissue preparation}

Sample collection was carried out as described previously[21]. First trimester villous and placental (maternal side) tissues were harvested within 1 hour of delivery. After collection, tissues taken from villous and placenta were frozen and stored at $-80^{\circ} \mathrm{C}$ or fixed in formaldehyde (4\%) and then embedded in paraffin for immunohistochemistry (IHC) studies. Fresh first trimester villous were rinsed in PBS prior to explant culture.

\section{Reagents}

17- $\beta$-estradiol (E2) was purchased from Abcam (Cambridge, MA, USA), G1 and G15 were purchased from Sigma ( St. Louis, MO, USA).

\section{Cell culture and treatments}


The immortalized human trophoblast cell line HTR8/SVneo was kindly provided by Dr. C. H. Graham (Queen's University, Kingston, ON, Canada). The cells were cultured in Medium-1640 (GIBCO, Invitrogen, Carlsbad, CA, USA), supplemented with $10 \%$ (v/v) fetal bovine serum (GIBCO, Australia) at $37^{\circ} \mathrm{C}$ in $5 \% \mathrm{CO}_{2}$ in air. $\mathrm{HTR} 8 / \mathrm{SVneo}$ were treated with E2 $(0.1 \mu \mathrm{M}), \mathrm{G} 1(0.01 \mu \mathrm{M})$ and/or $\mathrm{G} 15(0.1 \mu \mathrm{M})$, and then incubated in $5 \% \mathrm{CO}_{2}$ incubator (Thermo Fisher Scientific, Basingstoke, UK) for 24 hours, $0.1 \%$ DMSO was used as the control (CON).

\section{Explant culture and treatments}

The explant culture was performed as described previously[22] . In brief, 2-3 mm placental villous tips were dissected from first trimester human placentas $(6 \mathrm{w}-10 \mathrm{w})$ and cultured in 48-well culture dishes, which were pre-coated with phenol red-free matrigel substrate (Becton Dickinson, Bedford, MA, USA). The explants were cultured for 4 hours in 1640 medium supplemented with 20\% FBS, $100 \mathrm{IU} / \mathrm{ml}$ penicillin and $100 \mathrm{mg} / \mathrm{ml}$ streptomycin. Once the explants anchored on matrigel and started to outgrow, different treatments were administered, and explants incubated in $5 \% \mathrm{CO}_{2}$ incubator. The distance EVT cells had migrated was assessed after 24 hours.

\section{Immunohistochemistry (IHC)}

IHC was performed as reported previously. Briefly, $5 \mu \mathrm{m}$ thick paraffin embedded tissue sections were deparaffinized in xylene, rehydrated in a serial gradient of ethanol and then rinsed in PBS. Tissue sections were then quenched sequentially by using $3 \%$ hydrogen peroxide for 15 min and incubated in $10 \%$ normal goat serum (Sigma, St Louis, MO, USA) for 1 hour at room temperature. The slides were then incubated at $4^{\circ} \mathrm{C}$ overnight with polyclonal rabbit anti-GPR30 antibody (1:300, Abcam, ab39742) or polyclonal rabbit anti-cytokeratin7 (CK7) antibody (1:300, Abcam, ab68459). The slides were then rinsed with PBS, and further incubated with horseradish peroxidase-conjugated goat anti-rabbit IgG for 1 hour at $37^{\circ} \mathrm{C} \cdot 3,3^{\prime}-$ diaminobenzidine (DAB; chromogenic reagent, ZSGB-BIO, China) was used as the chromogen, and hematoxylin (Sigma) was used as a nuclear counterstain. Negative controls were prepared by omitting primary antibodies. The Image-Pro Plus software (version 6.0, NIH image) was used to measure the integrated optical density values; 5 view fields were randomly selected for each slide, and experiments were repeated in triplicate.

\section{Western blotting}


Western blotting was performed according to the approach previously established in our laboratory[21, 23]. After treatment, proteins were extracted from HTR8/SVneo cells for Western blotting. The primary antibodies used were anti-GPR30 (1:2000, Abcam, ab154069), anti-MMP9 (1:1000, CST, \#3852), anti-p-PI3K (1:1000, CST, \#4228), anti-PI3K (1:1000, CST, \#5405), anti-p-Akt (1:1000, CST, \#9271), anti-Akt (1:1000, CST, \#9272) and anti- $\beta$-actin (1:1000, ZSGB-BIO, TA-09).

\section{Transwell cell invasion assay}

Transwell cell invasion assays were performed as reported previously[24]. In brief, 24-well plates were used as the outer chambers and polycarbonate filters (8- $\mu \mathrm{m}$ pores, Transwell chamber, Millipore) were used as the inner chambers. The inner chambers were coated with $70 \mathrm{ml}$ of $10 \mu \mathrm{g} / \mathrm{ml}$ matrigel for 2 hours at $37^{\circ} \mathrm{C}$. $1.0 \times 10^{5}$

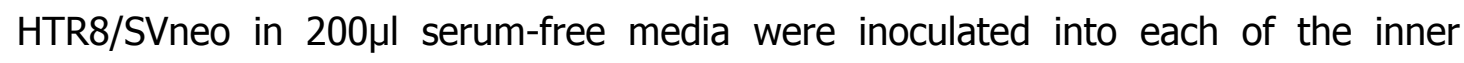
chambers and $700 \mu \mathrm{l}$ medium supplemented with $10 \%$ FBS was added into the outer chamber of each well. After 24 hours of incubation at $37^{\circ} \mathrm{C}$, the cells on the upper surface of the filter were completely removed by gently scraping with a cotton swab, and the invasive cells were fixed with $90 \%$ methanol for 10 min and then stained with $0.5 \%$ crystal violet. A light microscope (IX51; Olympus, Japan) was used to count the number of migrated cells, and the experiment was performed in triplicate.

\section{Immunofluorescence (IF)}

The immunofluorescence staining of GPR30 in HTR8/SVneo was performed as previously described[24]. After treatment, the cells were fixed with formaldehyde (4\%) and blocked with $10 \%$ normal goat serum (Sigma, St. Louis, MO, USA), then

incubated with anti-GPR30 antibody for 48 hours (1:250, Abcam, ab154069). A fluorescein isothiocyanate-conjugated goat anti-rabbit antibody (1:50 Santa Cruz Biotechnology, CA, USA) was then applied for 1 hour at room temperature. Presidium iodide $(3 \mathrm{mg} / \mathrm{mL})$ was used as a nuclear stain. Images were acquired by confocal microscopy (FV10i; Olympus, Japan).

\section{Statistical analysis}

All the data are expressed as mean \pm standard deviation (SD). The statistical analyses were performed with GraphPad Prism software (version 5.0). $\quad p<0.05$ was considered statistically significant.

\section{Results}




\section{Clinical characteristics}

Clinical data from all the subjects are summarized in Table 1. All the subjects were similar in maternal age, body mass index (BMI), nulliparity, length of gestation and all denied having a history of smoking. The women complicated with PE had significantly higher mean arterial pressure (MAP), 24-hours proteinuria and significantly lower neonatal birth weight and placental weight.

\section{PE is associated with compromised placental GPR30 expression.}

IHC staining of placental villi demonstrated that GPR30 is highly expressed in the outer trim of villi of first trimester placentas ( 6 weeks and 10 weeks of conception). Further investigation, using CK7 staining, identified these cells as extravillous trophoblasts (Fig.1). GPR30 expression was then investigated in term placenta. Intriguingly, semi-quantification of IHC staining demonstrated that GPR30 expression was significantly higher in term placentas from the gestational matched control (GMC) compared with the PE group (Fig.2, A, B). And this has been further confirmed by western blot, which shows that GPR30 expression was reduced more than $35 \%$ in PE placentas compare to GMC group. (Fig.2, C, D)

\section{HTR8/SVneo cells invasion is altered by pharmacological modulations of GPR30}

Immunofluorescent staining was initially performed to determine GPR30 expression in HTR8/SVneo cells. The result suggested that GPR30 was highly expressed in HTR8/SVneo cells(Fig.3A); this was further confirmed by western blots (Fig.3B).

As show in Fig.4, the invasive capability of HTR8/SVneo cells was enhanced about 2 fold by the treatments of E2 or GPR30 specific activator G1, while the effects of E2 were totally blocked by the addition of G15, a specific GPR30 inhibitor.

\section{GPR30 upregulates PI3K/Akt pathway and MMP9 expression in HTR8/SVneo cells}

Immunobloting demonstrated that basal GPR30 expression was very low in HTR8/SVneo cells, but was gradually upregulated by extended incubation with E2. 
The upregulation of GPR30 protein expression by E2 occurred in a time-dependent manner, and the highest GPR30 expression was observed after 24 hours of E2 incubation (Fig.5A, B). Surprisingly, the changes in MMP9 expression in HTR8/SVneo cells were similar to those observed with GPR30 in response to E2 treatment (Fig.5C). To further confirm the regulatory effects of GPR30 on MMP9 expression, E2, G1 and G15 were applied to HTR8/SVneo cells; both of E2 and G1 increased MMP9 expression, although G15 didn't show significant inhibitory effects on MMP9 expression (Fig.6A).

In addition, treating HTR8/SVneo cells with E2 or G1 increases phosphorylation of PI3K, and this effect was antagonized by treatment with G15 (Fig.6B). In consistent, modulation of GPR30 demonstrated similar effects on PI3K downstream AKt phosphorylation. (Fig.6C).

\section{Estradiol induced outgrowth in placental extravillous explants is mediated by GPR30}

To validate our findings from the in vitro cell model, the effects of pharmacological modulation of GPR30 on villous explant outgrowth have been further evaluated.

As shown in Fig.7 (A-E), treatment with either E2 or G1 resulted in an increase in explant migration distance compared to controls. However, additional G15 counteracts E2 induced trophoblast migration, with notable disarrangement of trophoblast cells at the rims of villous explants. GPR30 expression in the explants was confirmed by IF staining; the expression pattern was similar to that seen in the cell model (Fig.7a-e).

\section{Discussion}

PE is a life threatening pregnancy complication associated with multi-system disorders[25]. Despite extensive research, the etiology and pathogenesis of PE have not yet been fully elucidated. Accumulating evidence, however, has demonstrated that compromised trophoblast invasion is associated with PE. It is known that trophoblasts invade the uterus and maternal spiral arteries during the first trimester of pregnancy, resulting in decidulization and remodeling of the spiral arteries, effects 
that are critical for maintaining normal pregnancy. Insufficient invasion of trophoblast cells into decidua contributes to shallow placental implantation and inadequate conversion of the uterine spiral arteries $[4,7,10,11,26]$, which in turn will lead to poor perfusion of the placenta, and $H / R$ injury in placenta cells, all of which have been associated with the development of $\operatorname{PE}[3,11,27]$. Trophoblast cells have been compared with cancer cells, due to their similar invasive phenotype[18, 19]. There are, however, some important differences. For instance, although trophoblast cells are highly invasive in the early stage of pregnancy, this invasive phenotype gradually declines to avoid excessive invasion of placental tissue in the uterus, as observed in e.g. retained placenta. This differs to cancer cells, which has persistent invasiveness.

Various theories have been proposed regarding the possible changes to the regulatory mechanism(s) of trophoblast cell invasion resulting in PE. These include increased inflammation, a natural killer cell mediated immune response, and nutritional imbalance[28-31]. However, the possible role of hormones in regulating trophoblast cell invasion, and therefore their possible contribution to the development of PE, has not been fully understood.

Steroid hormones are involved in regulating various cellular events by activating a receptor family of transcription factors. The concentration of estrogens dramatically increases following conception and reaches a peak at term, indicating it is vital for gestation[32]. Estrogens influence gene expression, growth and cellular differentiation in target tissues by activating estrogen receptors. Intriguingly, estriol and estradiol concentrations are significantly lower in placental tissue from PE complicated pregnancies[33]. A liquid chromatography mass spectrometry study demonstrated that an aberrant plasma level of estrogen was associated with $\mathrm{PE}$; estradiol-17 $\beta$ was significantly lower in women with both mild PE (MPE) and severe PE (SPE), while estriol was only lower in cases SPE [34]. In addition, the circulating level of an estrogen metabolite, 2-Methoxyestradiol (2-ME) is significantly upregulated in normal pregnancy when compared to the menstrual cycle. However, plasma levels of 2-ME are significantly reduced in women with PE[35]. This is consistent with animal studies that demonstrated that a deficiency in catechol-Omethyl transferase, and a subsequent reduction in 2-ME concentration, resulted in 
the development of PE-like symptoms[36]. Taken together, this evidence supports the hypothesis that estrogens play an important role in PE development.

Estrogens trigger numerous transduction signaling pathways through its classical receptors ER a and ER $\beta$. It has been reported that elevated ER a mRNA and protein levels are associated with SPE [37], and ER $\beta$ is also significantly elevated in trophoblast cells of placentas from women with PE [38]. A novel estrogen receptor, GPR30 has been shown to play a significant role in mediating estrogen-induced invasion in various cancer cells[15, 39, 40]. In particular, it has been noted that GPR30 mediates 17- $\beta$-estradiol induced migration and invasion of breast cancer cells[41, 42]. Therefore, it has been proposed that GPR30 is involved in regulating trophoblast invasion by mediating the effects of estrogen or its metabolites[19]. However, solid evidence demonstrating the ability of GPR30 to modulate placental trophoblast cell invasion remains elusive.

In the present study, we are the first to report GPR30 expression in placenta, specifically in trophoblast cells, through out the entire course of pregnancy. This indicates that GPR30 could play a very important role in maintaining normal pregnancies, and the predominant localization of GPR30 in trophoblast cells implies it may be critical for trophoblast function.

In addition, placentas collected at term from pregnancies complicated by $\mathrm{PE}$ demonstrated a dramatic reduction in GPR30 expression, suggesting this might be a causative factor in the pathogenesis of $\mathrm{PE}$, although larger sample size and additional ethnical groups should be included for further validating this finding. The mechanisms that underlying the reduction of GPR30 in PE placenta haven't been fully elucidated by this study, however, the most recent work has shown that the ratelimiting enzyme of estrogen production from androgen, aromatase, is compromised in PE placenta[43], which is consistent with previous finding that estradiol expression is repressed in PE [37]. In the present study, we demonstrated that GPR30 expression could be induced by estradiol treatment in trophoblast (Fig5A, B). Taken together, this information implies that the down-regulation of placental GPR30 in PE could be attribute to aberrant estrogen synthesis. 
Considering that trophoblast invasion is dramatically reduced in term placenta compared to first trimester, and that shallow trophoblast invasion primarily occurs in early pregnancy, term placenta is not ideal for studying trophoblast invasion. Thus, in this study we utilized an immortalized human trophoblast cell line and primary first trimester villi explants to investigate the effects of GPR30 on trophoblast invasion.

Pharmacological manipulations of GPR30 activity have been applied to a number of in vitro models including primary placental explants and an immortalized human trophoblast cell line (HTR8/SVneo), both of which are well established models used to study trophoblast invasion[44]. Our data demonstrated that the invasive capacity of explants and HTR8/SVneo cells could be significantly up-regulated by both the selective GPR30 agonist G1 and a general estrogen receptor agonist E2. The effects of $\mathrm{G} 1$ and E2 can be subsequently abolished by co-incubation with the selective GPR30 inhibitor G15. These results strongly support the hypothesis that GPR30, rather than the classical ERs, plays a major role in mediating estrogen-regulated trophoblast invasion. However, G15 treatment alone failed to result in a significant reduction in trophoblast invasion compared with control; this may be due to relatively low basal activity of GPR30 in HTR8/SVone cells. Alternatively, the adaptive signaling pathways that are also involved in regulating trophoblast invasion may have compensated for the loss of GPR30 function.

It is well known that migratory trophoblast cells express MMPs, cathepsins, and urokinase plasminogen activator, which are able to degrade extracellular matrix (ECM) of uterus tissue, and thus facilitate extravillous trophoblast (EVT) invasion [45, 46]. To further elucidate the mechanisms that underlie GPR30-mediated trophoblast invasion, we investigated the effects of GPR30 on the expression of MMPs. We found that treatment with both E2 and G1 was able to induce MMP9 expression in HTR8/SVone cells, but that co-incubation with G15 dramatically inhibited MM9 protein expression. These data support the proposal that MMP9 is a downstream effector of GPR30.

Numerous cancer studies have shown that the PI3K/Akt pathway promotes cancer cell invasion and tumor metastasis by upregulating the expression of $\operatorname{MMPs}[47-49]$. 
To further validate whether the effects of GPR30 on trophoblast invasion were mediated by PI3K/Akt-MMP9, we determined the effect of GPR30 agonists on the PI3K/Akt signaling pathway. Our data elucidated that both E2 and G1 treatment significantly augmented the phosphorylation levels of both PI3K and Akt, while such activation of PI3K/Akt pathway was attenuated by G15 treatment. The response of PI3K/Akt to GPR30 modulation is in accordance with increased MMP9 expression, suggesting PI3K/Akt could be coupled with MMP9 expression in trophoblast cells to mediate GPR30-regulated cell invasion.

In conclusion, the present study demonstrates that GPR30 is expressed in human trophoblast cells and its expression is significantly impaired in PE. Moreover, pharmacological modulation of GPR30 is able to regulate HTR8 invasion in vitro, and such effects may be mediated by the PI3K/Akt-MMP9 signaling axis. Therefore, modulation of GPR30 could be promising for benefiting PE and other pregnancy complications associated with impaired trophoblast invasion.

\section{Acknowledgments}

This study was supported by the National Natural Science Foundation of China (No. 81170585, 81100444, 81370732), Chongqing Science and Technology Commission (No. 2011BB5121) and Chongqing Municipal Health Bureau (No.2011-2-046). 
Table 1

Clinical characteristics of the two groups

\begin{tabular}{lll}
\hline Category & Preeclampsia group & Normotensive group \\
\hline Number & 21 & 20 \\
Age (years) & $28.04 \pm 3.05$ & $27.69 \pm 3.12$ \\
Length of gestation (weeks) & $35.48 \pm 1.65$ & $37.92 \pm 0.98$ \\
Nulliparity (\%) & $64.66 \%$ & $80 \%$ \\
MAP (mm Hg) & $118.71 \pm 5.14 *$ & $85.90 \pm 3.60$ \\
24-Hour proteinuria $(\mathrm{g})$ & $2.09 \pm 0.56 *$ & $0.08 \pm 0.03$ \\
Smoking history & None & None \\
BMI (kg/m2) & $24.01 \pm 2.60$ & $24.70 \pm 2.34$ \\
Neonatal birth weight $(\mathrm{g})$ & $2493.04 \pm 246.19 *$ & $3016.49 \pm 320.98$ \\
Placental weight $(\mathrm{g})$ & $454.28 \pm 40.07 * *$ & $542.45 \pm 53.72$
\end{tabular}

$* p<0.01, * * p<0.05$ 


\section{Figure legends}

Fig.1: GPR30 is expressed in first trimester human placental villi.

(A) Immunohistochemistry (IHC) of GPR30 in first trimester villous tissue at different gestational ages. Cytokeratin 7 (CK7) staining labels extravillous trophoblast cells. Bars: $100 \mu \mathrm{m}$. Insets: Higher magnification views. 5 view fields were randomly selected from each sample (B) Quantification of GPR30 IHC staining of first trimester villous tissue. 
Fig.2: GPR30 is expressed in third trimester human placental tissue.

(A) Immunostaining of GPR30 in villi of term placenras, from pregnancies complicated by PE and gestational matched control (GMC). CK7 staining demonstrates extravillous trophoblast cells. Negative controls were prepared by omitting the primary antibody. Bars: $100 \mu \mathrm{m}$. Insets: Higher magnification views (B) Semi-quantitative immunohistochemistry analysis of GPR30 expression in placental tissue, 5 view fields were randomly selected from each sample $\left({ }^{*} p<0.001, \mathrm{n}=3\right)$. (C) Representative Western blots of GPR30 in third trimester placental tissue from cases of PE and GMC. $\beta$-actin was used as a loading control (here and after); (D) GPR 30 protein expression quantification of PE and GMC placentas ( $*^{*} p<0.01, \mathrm{n}=3$ ). 
Fig.3: GPR30 expressed in HTR8/SVneo cells.

(A) Confocal microscopic images of GPR30 immunofluorescent staining in HTR8/SVneo cells. The primary antibody was omitted in the negative control panel.

Bars: $75 \mu \mathrm{m}$, insets: higher magnification views. (B) Immunoblot of GPR30 in HTR8/SVneo cells. 
Fig.4: GPR30 activation promotes HTR8/SVneo cell invasion.

(A-E) Representative images of filters containing cells from the matrigel invasion assay are shown (A) Controls; (B) E2 treatment; (C) G1 treatment; (D) G15 treatment; (E) $\mathrm{E} 2+\mathrm{G} 15$ treatment; $(\mathrm{F})$ Quantification of transwell assay ( ${ }^{*} p<0.01 \mathrm{vs.}$ CON, $\# p<0.01$ vs. E2); Experiment was repeated 3 times. 
Fig.5: E2 treatment increases GPR30 and MMP9 expression in HTR8/SVneo cells in a time-dependent manner.

(A) Representative Western blots of GPR30 and MMP9 in HTR8/SVneo cells in response to a time course E2 treatment; (B) Densitometry analysis of GPR30 Western bands ( $* p<0.001$ vs.0h; $\# p<0.0001$ vs.0h); (c) Densitometry analysis of MMP9 Western bands ( ${ }^{*} p<0.05$ vs.0h; $\# p<0.001$ vs.0h); Experiment was repeated 3 times. 
Fig.6: MMP9 expression and PI3K/Akt activation is increased in response to GPR30 modulation in HTR8/SVneo cells.

(A) Representative Western blots of MMP9 in response to E2, G1 and G15 treatments ( ${ }^{*} p<0.001$ vs. CON; $\# p<0.001$ vs. CON); (B) PI3K-phosphorylation in response to $\mathrm{E} 2, \mathrm{G} 1$ and $\mathrm{G} 15$ treatments (* $p<0.05$ vs. CON; $\# p<0.01$ vs. CON); (C) Akt-phosphorylation in response to $\mathrm{E} 2, \mathrm{G} 1$ and $\mathrm{G} 15$ treatments ( $* p<0.05$ vs. CON; $\# p<0.01$ vs. CON; $\S p<0.01$ vs. E2). The densitometry analysis was normalized by $\beta$-actin, experiment was repeated 3 times. 
Fig.7: GPR30 on extravillous trophoblast outgrowth in vitro.

(A-E) Extravillous explants were cultured on matrigel, serial pictures of explants incubated with different treatments were taken using a light microscope. Bars: 100 $\mu \mathrm{m}$. (a-e):Immunofluorescent staining of GPR30 in villous explants. Bars: $100 \mu \mathrm{m}$. (A, a) CON; (B, b) E2; (C, C) G1; (D, d) G15; (E, e) E2+G15; (F) Quantification of villous explants migration distance ( ${ }^{*} p<0.05$ vs. CON, $\# p<0.05$ vs. E2). 5 view fields were randomly selected from each treatment. 


\section{References}

1. Sibai BM. Diagnosis and management of gestational hypertension and preeclampsia. Obstetrics and gynecology. 2003; 102 (1):181-92.

2. Cunningham FG, Lindheimer MD. Hypertension in pregnancy. The New England journal of medicine. 1992; 326 (14):927-32.

3. Pennington KA, Schlitt JM, Jackson DL, Schulz LC, Schust DJ. Preeclampsia: multiple approaches for a multifactorial disease. Disease models \& mechanisms. 2012; 5 (1):9-18.

4. Reister F, Frank HG, Kingdom JC, Heyl W, Kaufmann P, Rath W, et al. Macrophage-induced apoptosis limits endovascular trophoblast invasion in the uterine wall of preeclamptic women. Laboratory investigation; a journal of technical methods and pathology. 2001; 81 (8):1143-52.

5. Khong TY, De Wolf F, Robertson WB, Brosens I. Inadequate maternal vascular response to placentation in pregnancies complicated by pre-eclampsia and by small-for-gestational age infants. British journal of obstetrics and gynaecology. 1986; 93 (10):1049-59.

6. Huppertz B. Placental origins of preeclampsia: challenging the current hypothesis. Hypertension. 2008; 51 (4):970-5.

7. Redman CW. Current topic: pre-eclampsia and the placenta. Placenta. 1991; $12(4): 301-8$.

8. Brosens IA, Robertson WB, Dixon HG. The role of the spiral arteries in the pathogenesis of preeclampsia. Obstetrics and gynecology annual. 1972; 1:177-91.

9. Shah DA, Khalil RA. Bioactive factors in uteroplacental and systemic circulation link placental ischemia to generalized vascular dysfunction in hypertensive pregnancy and preeclampsia. Biochemical pharmacology. 2015; 95 (4):211-26.

10. Goldman-Wohl D, Yagel S. Regulation of trophoblast invasion: from normal implantation to pre-eclampsia. Molecular and cellular endocrinology. 2002; 187 (12):233-8.

11. Burton GJ, Woods AW, Jauniaux E, Kingdom JC. Rheological and physiological consequences of conversion of the maternal spiral arteries for uteroplacental blood flow during human pregnancy. Placenta. 2009; 30 (6):473-82.

12. Olde B, Leeb-Lundberg LM. GPR30/GPER1: searching for a role in estrogen physiology. Trends in endocrinology and metabolism: TEM. 2009; 20 (8):409-16. 13. Prossnitz ER, Arterburn JB, Smith HO, Oprea TI, Sklar LA, Hathaway HJ. Estrogen signaling through the transmembrane $\mathrm{G}$ protein-coupled receptor GPR30. Annual review of physiology. 2008; 70:165-90.

14. Lappano R, Pisano A, Maggiolini M. GPER Function in Breast Cancer: An Overview. Frontiers in endocrinology. 2014; 5:66.

15. Scaling AL, Prossnitz ER, Hathaway HJ. GPER mediates estrogen-induced signaling and proliferation in human breast epithelial cells and normal and malignant breast. Hormones \& cancer. 2014; 5 (3):146-60.

16. Petrie WK, Dennis MK, Hu C, Dai D, Arterburn JB, Smith HO, et al. G proteincoupled estrogen receptor-selective ligands modulate endometrial tumor growth. Obstetrics and gynecology international. 2013; 2013:472720.

17. Maggiolini M, Picard D. The unfolding stories of GPR30, a new membranebound estrogen receptor. The Journal of endocrinology. 2010; 204 (2):105-14. 18. Fisher SJ, Damsky CH. Human cytotrophoblast invasion. Seminars in cell biology. 1993; 4 (3):183-8.

19. Knofler M, Pollheimer J. IFPA Award in Placentology lecture: molecular regulation of human trophoblast invasion. Placenta. 2012; 33 Suppl:S55-62. 
20. Practice ACoO. ACOG practice bulletin. Diagnosis and management of preeclampsia and eclampsia. Number 33, January 2002. American College of Obstetricians and Gynecologists. International journal of gynaecology and obstetrics: the official organ of the International Federation of Gynaecology and Obstetrics. 2002; 77 (1):67-75.

21. Luo X, Yao ZW, Qi HB, Liu DD, Chen GQ, Huang S, et al. Gadd45alpha as an upstream signaling molecule of p38 MAPK triggers oxidative stress-induced sFlt-1 and sEng upregulation in preeclampsia. Cell and tissue research. 2011; 344 (3):55165 .

22. Genbacev O, Jensen KD, Powlin SS, Miller RK. In vitro differentiation and ultrastructure of human extravillous trophoblast (EVT) cells. Placenta. 1993; 14 (4):463-75.

23. Tong C, Morrison A, Mattison S, Qian S, Bryniarski M, Rankin B, et al. Impaired SIRT1 nucleocytoplasmic shuttling in the senescent heart during ischemic stress. FASEB journal : official publication of the Federation of American Societies for Experimental Biology. 2013; 27 (11):4332-42.

24. Yang Z, Bai B, Luo X, Xiao X, Liu X, Ding Y, et al. Downregulated Kruppel-like factor 8 is involved in decreased trophoblast invasion under hypoxia-reoxygenation conditions. Reproductive sciences. 2014; 21 (1):72-81.

25. Redman CW, Sargent IL. Latest advances in understanding preeclampsia. Science. 2005; 308 (5728):1592-4.

26. Pijnenborg R, Anthony J, Davey DA, Rees A, Tiltman A, Vercruysse L, et al. Placental bed spiral arteries in the hypertensive disorders of pregnancy. British journal of obstetrics and gynaecology. 1991; 98 (7):648-55.

27. Hung TH, Burton GJ. Hypoxia and reoxygenation: a possible mechanism for placental oxidative stress in preeclampsia. Taiwanese journal of obstetrics \& gynecology. 2006; 45 (3):189-200.

28. Kalkunte $S$, Nevers T, Norris WE, Sharma S. Vascular IL-10: a protective role in preeclampsia. Journal of reproductive immunology. 2011; 88 (2):165-9.

29. Redman CW, Sargent IL. Immunology of pre-eclampsia. American journal of reproductive immunology. 2010; 63 (6):534-43.

30. Cotechini T, Komisarenko M, Sperou A, Macdonald-Goodfellow S, Adams MA, Graham $\mathrm{CH}$. Inflammation in rat pregnancy inhibits spiral artery remodeling leading to fetal growth restriction and features of preeclampsia. The Journal of experimental medicine. 2014; 211 (1):165-79.

31. Newman V, Fullerton JT. Role of nutrition in the prevention of preeclampsia. Review of the literature. Journal of nurse-midwifery. 1990; 35 (5):282-91.

32. Torrealday S, Taylor HS, Burney RO, Mooney SB, Giudice LC. Endocrinology of Pregnancy. In: De Groot LJ, Beck-Peccoz P, Chrousos G, Dungan K, Grossman A, Hershman JM, et al., (editors). Endotext. South Dartmouth (MA); 2000.

33. Acikgoz S, Bayar UO, Can M, Guven B, Mungan G, Dogan S, et al. Levels of oxidized LDL, estrogens, and progesterone in placenta tissues and serum paraoxonase activity in preeclampsia. Mediators of inflammation. 2013; 2013:862982.

34. Jobe SO, Tyler CT, Magness RR. Aberrant synthesis, metabolism, and plasma accumulation of circulating estrogens and estrogen metabolites in preeclampsia implications for vascular dysfunction. Hypertension. 2013; 61 (2):480-7.

35. Perez-Sepulveda A, Espana-Perrot PP, Norwitz ER, Illanes SE. Metabolic pathways involved in 2-methoxyestradiol synthesis and their role in preeclampsia. Reproductive sciences. 2013; 20 (9):1020-9. 
36. Kanasaki K, Palmsten K, Sugimoto H, Ahmad S, Hamano Y, Xie L, et al. Deficiency in catechol-O-methyltransferase and 2-methoxyoestradiol is associated with pre-eclampsia. Nature. 2008; 453 (7198):1117-21.

37. Yin G, Zhu X, Guo C, Yang Y, Han T, Chen L, et al. Differential expression of estradiol and estrogen receptor alpha in severe preeclamptic pregnancies compared with normal pregnancies. Molecular medicine reports. 2013; 7 (3):981-5.

38. Schiessl B, Mylonas I, Hantschmann P, Kuhn C, Schulze S, Kunze S, et al. Expression of endothelial NO synthase, inducible NO synthase, and estrogen receptors alpha and beta in placental tissue of normal, preeclamptic, and intrauterine growth-restricted pregnancies. The journal of histochemistry and cytochemistry : official journal of the Histochemistry Society. 2005; 53 (12):1441-9.

39. Ohshiro K, Schwartz AM, Levine PH, Kumar R. Alternate estrogen receptors promote invasion of inflammatory breast cancer cells via non-genomic signaling. PloS one. 2012; 7 (1):e30725.

40. Ge X, Guo R, Qiao Y, Zhang Y, Lei J, Wang X, et al. The G protein-coupled receptor GPR30 mediates the nontranscriptional effect of estrogen on the activation of PI3K/Akt pathway in endometrial cancer cells. International journal of gynecological cancer : official journal of the International Gynecological Cancer Society. 2013; 23 (1):52-9.

41. Weissenborn C, Ignatov $T$, Poehlmann A, Wege AK, Costa SD, Zenclussen AC, et al. GPER functions as a tumor suppressor in MCF-7 and SK-BR-3 breast cancer cells. Journal of cancer research and clinical oncology. 2014; 140 (4):663-71.

42. Wei W, Chen ZJ, Zhang KS, Yang XL, Wu YM, Chen XH, et al. The activation of $\mathrm{G}$ protein-coupled receptor 30 (GPR30) inhibits proliferation of estrogen receptornegative breast cancer cells in vitro and in vivo. Cell death \& disease. 2014; 5:e1428. 43. Perez-Sepulveda A, Monteiro LJ, Dobierzewska A, Espana-Perrot PP, VenegasAraneda P, Guzman-Rojas AM, et al. Placental Aromatase Is Deficient in Placental Ischemia and Preeclampsia. PloS one. 2015; 10 (10):e0139682.

44. Pollheimer J, Knofler M. Signalling pathways regulating the invasive differentiation of human trophoblasts: a review. Placenta. 2005; 26 Suppl A:S21-30. 45. Bischof $P$, Meisser A, Campana A. Paracrine and autocrine regulators of trophoblast invasion--a review. Placenta. 2000; 21 Suppl A:S55-60.

46. Knofler M. Critical growth factors and signalling pathways controlling human trophoblast invasion. The International journal of developmental biology. 2010; 54 (2-3):269-80.

47. Gao Y, Guan Z, Chen J, Xie H, Yang Z, Fan J, et al. CXCL5/CXCR2 axis promotes bladder cancer cell migration and invasion by activating PI3K/AKT-induced upregulation of MMP2/MMP9. International journal of oncology. 2015.

48. Dey JH, Bianchi F, Voshol J, Bonenfant D, Oakeley EJ, Hynes NE. Targeting fibroblast growth factor receptors blocks PI3K/AKT signaling, induces apoptosis, and impairs mammary tumor outgrowth and metastasis. Cancer research. 2010; 70 (10):4151-62.

49. Dennis MK, Burai R, Ramesh C, Petrie WK, Alcon SN, Nayak TK, et al. In vivo effects of a GPR30 antagonist. Nature chemical biology. 2009; 5 (6):421-7. 